\title{
Método empírico para estimar la densidad básica en muestras pequeñas de madera
}

\author{
Salvador Valencia Manzo ${ }^{1}$ \\ J esús Vargas Hernández ${ }^{2}$
}

\begin{abstract}
RESUMEN
Se evaluó un método empírico para calcular el volumen en muestras pequeñas de madera obtenidas con taladro de Pressler, que se utilizan en la estimación de la densidad básica de la madera y se comparó dicha estimación con otros métodos de uso común en la estimación de la densidad básica de la madera, como son el método de desplazamiento en agua y el método de máximo contenido de humedad. Los valores promedio, de desviación estándar, mínimo y máximo, de densidad de la madera obtenidos con el método empírico fueron muy semejantes a los obtenidos con los otros dos métodos, y la correlación simple que se presentó fue cercano a la unidad, por lo que el método empírico es altamente confiable, rápido y sencillo en la estimación de la densidad en muestras pequeñas de madera, que tienen forma geométrica regular.
\end{abstract}

PALABRAS CLAVE:

Densidad básica, peso específico, método empírico, taladro de Pressler, Pinus greggii

\begin{abstract}
An empirical method to calculate the volume of small samples of wood obtained with an increment borer, for the purpose of estimating specific gravity, was evaluated. The empirical method $w$ as compared with other methods commonly used: the w ater displacement method and the maximum moisture method. The mean values, standard deviation, specific gravity minimum and maximum obtained with the empirical method were significantly correlated with those values obtained with the other two methods. This indicates that the empirical method is a reliable option, as well as being faster and simpler, for estimating the specific gravity of small samples of regularly shaped wood.
\end{abstract}

KEY WORDS:

Specific gravity, specific w eight, empirical method, increment borer, Pinus greggii

1 Departamento Forestal. Universidad Autónoma Agraria Antonio Narro. 25315 Buenavista, Saltillo, Coah.

2 Programa Forestal. Colegio de Postgraduados. 56230 Montecillo, Edo. de México. Manuscrito recibido para su publicación el 9 de Abril de 1996 


\section{INTRODUCCION}

La densidad básica o relativa de la madera tiene un efecto importante sobre el rendimiento y calidad del producto final y se considera como la propiedad de la madera más importante para casi todos los productos maderables derivados de las especies forestales (Einspahr et al., 1969). A demás, la productividad total en biomasa de un rodal no puede determinarse a menos que se conozca la densidad promedio de la madera (Zobel y Talbert, 1984). Por otro lado, la densidad de la madera es una propiedad que muestra una amplia variación entre y dentro de especies, así como un fuerte control genético, por lo que es posible manipularla genéticamente (Zobel y Van Buijtenen, 1989). Por todo lo anterior, resulta de gran importancia desarrollar métodos rápidos, simples y precisos para la estimación de la densidad de la madera en especies forestales.

Se ha encontrado que la densidad promedio de la madera en un árbol puede representarse como una función del valor estimado en una muestra de madera tomada del árbol a una altura de $1.3 \mathrm{~m}$ (Higa, 1963); de manera general, la densidad promedio en todo el árbol es ligeramente menor que la densidad estimada en la muestra tomada a la altura del pecho (Ladrach, 1984). Para realizar la estimación de la densidad de la madera en un punto del árbol se han desarrollado procedimientos indirectos como el uso del "Pilodyn" (Cown, 1978) y del durómetro (Godfrey y Garthwaite, 1984) que proporcionan una rápida estimación de esta propiedad con base en la resistencia de la madera a la penetración del objeto (Sprague et al., 1983; Notivol et al., 1992), de manera que la estimación de la densidad corresponde a la parte más exterior del árbol y no representa necesariamente un valor promedio de toda la sección del fuste, dado que la densidad de la madera varía a lo largo y ancho del fuste (Zobel y Van Buijtenen, 1989). Además, la estimación de la densidad puede ser afectada por diversos factores al momento de realizar ésta.

Hasta ahora, la obtención de muestras de madera con taladro de Pressler es el método de estimación empleado con mayor frecuencia. La obtención de las virutas (cilindros) de madera permite estudiar varias características de la madera en forma simultánea, como la edad, el incremento anual, la densidad y las características de madera temprana y tardía en el anillo de crecimiento en diferentes edades del árbol, entre otras. Para la estimación de la densidad de la madera en estas muestras pequeñas existen varias técnicas, desde las más sencillas entre las que se tiene el método por desplazamiento de agua y el método de máximo contenido de humedad (Smith, 1954, 1955), hasta las más modernas como es el empleo de rayos-X (J ozsa y Brix, 1989).

El empleo de rayos-X para la estimación de la densidad de la madera es sumamente preciso, ya que proporciona un perfil de la variación en la densidad de la madera a lo largo de la muestra, pero requiere de un equipo relativamente más sofisticado. Con el método de máximo contenido de humedad, se requiere obtener el peso de la muestra de madera saturada y el peso anhidro de la misma, mientras que con el método de desplazamiento en agua la mayor dificultad se encuentra en estimar el volumen en verde de la muestra, dado su pequeño tamaño. Una forma de resolver este problema es considerar cada viruta como un cilindro perfecto con diámetro igual al diámetro interior del taladro que se utilice para la obtención de la muestra y una longitud igual al largo de la viruta que se obtenga; de este modo, si se emplea la fórmula para obtener el 
volumen de un cilindro, ya no será necesario utilizar el desplazamiento del agua, requerido en el método de desplazamiento en agua, ni obtener el peso de la muestra saturada requerido en el método de máximo contenido de humedad (Smith, 1954, 1955). El peso anhidro de la muestra necesario para estimar la densidad básica, se obtiene posteriormente al secar la muestra en estufa como en cualquiera de los métodos directos. Si se asume que la muestra de madera representa un cilindro, la estimación del volumen verde mediante la fórmula empírica resulta más sencilla y rápida y con una precisión similar a la de los otros métodos, lo que representa un ahorro considerable en tiempo cuando se realiza la estimación en una gran cantidad de muestras. Sin embargo, hasta ahora, no se han encontrado referencias sobre el uso o la comparación del método empírico con los otros métodos más utilizados comúnmente.

\section{OBJ ETIVO}

El objetivo de este trabajo es proponer el empleo del método empírico para calcular el volumen verde de muestras pequeñas (virutas) de madera en la estimación de la densidad básica de la madera, como una opción más y la cual se valida mediante comparación con otros dos métodos de uso común para estimar la densidad básica de la madera, como son el método de desplazamiento en agua y el método de máximo contenido de humedad.

\section{MATERIALES Y METODOS}

El material utilizado en el estudio se obtuvo de una plantación de Pinus greggii de 17 años de edad localizada a $7 \mathrm{~km}$ de Coatlinchán, Méx. Se seleccionaron 15 árboles de diferentes categorías diamétricas, procurando abarcar todas las categorías presentes. Se obtuvieron muestras de madera de lado a lado del tronco a una altura de $1.3 \mathrm{~m}$ de la superficie del suelo con un taladro Pressler de $5 \mathrm{~mm}$ de diámetro interior por $30 \mathrm{~cm}$ de largo; cada muestra se guardó en un popote de plástico para su traslado y almacenamiento en refrigerador hasta el momento de iniciar el trabajo en laboratorio, con el propósito de evitar su deshidratación. Cada muestra de madera (cilindro) se seccionó en dos partes a partir del centro del árbol, denominando sección $A$ a un lado y sección $B$ al lado opuesto y posteriormente se identificaron con tinta indeleble para evitar la confusión del material durante la etapa del laboratorio.

La estimación de la densidad básica de la madera se realizó por medio de tres métodos: a) el método empírico; b) el método de desplazamiento de agua; y c) el método de máximo contenido de humedad.

Las dos secciones se utilizaron para estimar la densidad de la madera por el método empírico. Adicionalmente, la sección $A$ se utilizó también para la estimación con el método de desplazamiento en agua y la sección $B$, para la estimación con el método de máximo contenido de humedad.

\section{a) Método empírico}

Para este método cada muestra de madera se consideró como un cilindro perfecto. Una vez seccionadas, y cuando se encontraban con un contenido de humedad por arriba del punto de saturación de la fibra, se midió únicamente el largo de cada sección utilizando una regla graduada con aproximación a milímetros. Con los valores de longitud de la muestra y del diámetro interior del taladro $(5 \mathrm{~mm})$ se obtuvo el volumen de cada sección de las 
muestras de madera, empleando:

$$
\mathrm{Vv}=3.1416 \mathrm{D}^{2} * \mathrm{~L} / 4
$$

donde:

$V v=$ volumen estimado del cilindro de madera $\left(\mathrm{cm}^{3}\right)$

$\mathrm{D}=$ diámetro interior del cilindro del taladro pressler $(0.5 \mathrm{~cm})$

$\mathrm{L}=$ largo de la muestra de madera (cm)

El peso anhidro (Po) de las muestras se obtuvo en una balanza analítica después de deshidratarse en una estufa a $105-110^{\circ} \mathrm{C}$ durante 24 horas. Esta estimación se realizó después de estimar el volumen verde de cada muestra por medio de los otros dos métodos, y sirvió para la estimación de la densidad de la madera en los tres métodos.

Con los datos de peso anhidro (Po) y volumen verde $(\mathrm{V} v)$ se obtuvo el valor de densidad básica de la madera (Db), según la fórmula:

$$
\mathrm{Db}=\mathrm{Po}_{\mathrm{O}} / \mathrm{Vv} \quad\left(\mathrm{g} / \mathrm{cm}^{3}\right)
$$

\section{b) Método de desplazamiento de agua}

Las muestras se colocaron en un recipiente con agua, donde permanecieron 7 días, hasta su saturación (peso constante). Para obtener el volumen por deplazamiento en agua se colocó un recipiente con agua sobre una balanza analítica (con precisión de 0.0001 g); enseguida se introdujo cada muestra de madera sin que tocara las paredes ni el fondo del recipiente, de modo que se obtuvo el peso del agua desplazada misma que corresponde al volumen de la muestra (Vv), considerando la densidad del agua como la unidad.

\section{c) Método de máximo contenido de humedad (MCH)}

Las muestras se colocaron en un recipiente con agua y se colocaron en una cámara de vacío para agilizar y lograr la penetración total del agua en la muestra. Después de 24 horas se pesaron en una balanza analítica (con precisión de 0.0001 g), quitando el exceso de agua; de este modo se obtuvo el peso saturado (Ps).

La fórmula utilizada para obtener la densidad de la madera con este método fue la siguiente, presentada por Smith (1954):

$$
D M=\frac{1}{M H C+\frac{1}{D b}}=\frac{1}{\frac{P S-P O}{P_{O}}+\frac{1}{D b}}
$$

Donde:

$\mathrm{Db}=$ densidad básica de la madera

$\mathrm{MHC}=$ máximo contenido de humedad en gramos de agua por gramos de madera seca $(\mathrm{g})$

Ps = peso de la muestra saturada $(\mathrm{g})$

Po = peso de la muestra anhidra $(\mathrm{g})$

$\mathrm{Dr}=$ densidad de la pared celular seca (1.53)

Las estimaciones de la densidad básica de la madera obtenidas con el método empírico se compararon con cada uno de los otros dos métodos mediante correlaciones simples para conocer la eficiencia del cálculo del volumen mediante el método empírico.

\section{RESULTADOS Y DISCUSION}

El valor promedio de la densidad básica de la madera estimado con el método empírico en cada una de las secciones de las muestras, es similar al valor medio estimado con cada uno de los otros métodos empleados para dicha sección (Tabla 1 ). 
Tabla 1. Comparación de valores de densidad básica de la madera $\left(\mathrm{g} / \mathrm{cm}^{3}\right)$ entre el método empírico (ME) y los métodos de desplazamiento de agua (MDA) y máximo contenido de humedad ( $\mathrm{MMCH}$ ).

\begin{tabular}{||c|c|c|c|c|c|c||}
\hline \hline SECCION & METODO & $\mathrm{N}$ & MEDIA & $\begin{array}{c}\text { DESVIACION } \\
\text { ESTANDAR }\end{array}$ & $\begin{array}{c}\text { VALOR } \\
\text { MINIMO }\end{array}$ & $\begin{array}{c}\text { VALOR } \\
\text { MAXIMO }\end{array}$ \\
\hline $\mathrm{A}$ & $\mathrm{MDA}$ & 15 & 0.418 & 0.023 & 0.382 & 0.453 \\
\hline $\mathrm{A}$ & $\mathrm{ME}$ & 15 & 0.420 & 0.023 & 0.385 & 0.463 \\
\hline $\mathrm{B}$ & $\mathrm{MMCH}$ & 15 & 0.427 & 0.032 & 0.393 & 0.503 \\
\hline $\mathrm{B}$ & $\mathrm{ME}$ & 15 & 0.426 & 0.032 & 0.391 & 0.506 \\
\hline
\end{tabular}

De igual modo, en los dos casos, el método empírico presentó básicamente la misma desviación estándar y valores mínimos y máximos similares a los obtenidos con los otros dos métodos. Lo anterior indica, que el método empírico puede tener una precisión similar a los otros métodos para estimar la densidad de la madera en muestra pequeñas tomadas con taladro de Pressler.

La correlación simple entre los valores de densidad de la madera obtenidos por medio del método empírico y los valores del método de desplazamiento en agua fue de $r=0.96$, mientras que la correlación de los valores del método empírico con los valores del método de máximo contenido de humedad fue de $r=0.99$ (Fig. 1), en ambos casos con una alta significancia estadística ( $P$ \#0.01).

Los altos valores de las correlaciones (cercanas a la unidad) del método empírico con los otros dos métodos (desplazamiento en agua y máximo contenido de humedad) muestran que el método empírico puede emplearse confiablemente para calcular el volumen de las muestras de madera obtenidas con taladro de Pressler, en una forma más rápida y sencilla en comparación con el procedimiento empleado en los métodos de desplazamiento en agua y de máximo contenido de humedad, lo que permite estimar más rápidamente la densidad de la madera y procesar mayor número de muestras. Estos mismos resultados se han comprobado en muestras de madera obtenidas de una plantación de progenies de Pinus patula donde se compararon los valores de densidad de la madera, obtenidos con el método empírico y el método de máximo contenido de humedad, en 48 árboles de 23 familias de polinización libre, en los cuales se obtuvieron prácticamente los mismos valores de densidad de la madera con ambos métodos, con una correlación de $r=0.99$ entre dichos valores, lo que permitió emplear el método empírico en la estimación del control genético de la densidad de la madera de dicho ensayo con el consiguiente ahorro de tiempo en el procesamiento de las muestras (Valencia, 1994). 


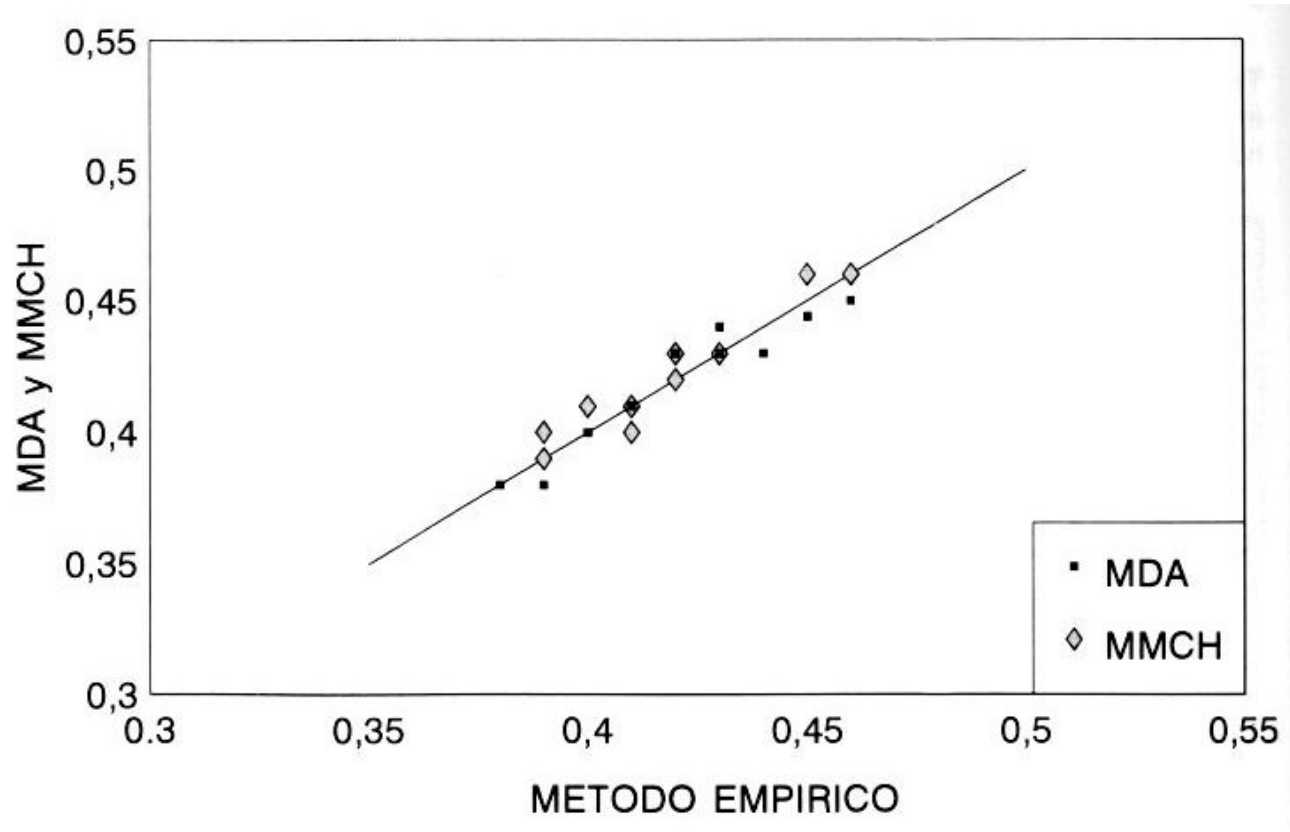

Figura 1. Valores de densidad de la madera estimados con el método empírico (ME) con respecto a los valores obtenidos con los métodos de desplazamiento en agua (MDA) y máximo contenido de humedad $(\mathrm{MCH})$.

\section{CONCLUSIONES}

El método empírico es altamente confiable en la estimación de la densidad en muestras pequeñas de madera, que tienen forma geométrica regular. En la práctica, el método empírico fue el método más sencillo, siguiéndole el método de máximo contenido de humedad y después el método de desplazamiento en agua.

\section{RECONOCIMIENTOS}

Este trabajo se realizó gracias al apoyo del Consejo Nacional de Ciencia y Tecnología (CONACYT), a través del proyecto 0425A-9108.

\section{REFERENCIAS}

Cown, D.J. 1978. Comparison of the pilodyn and tensiometer methods for the rapid assesment of wood density in living trees. N.Z.J. For. Sci. 8:384-391.

Einspahr, D.W., J .P. Van Buijtenen y J .R. Peckham. 1969 . Pulping characteristics of ten-year loblolly pine selected for extreme wood specific gravity. Sil. Gen. 18(3):57-61.

Godfrey, G.A. y D.K. Garthw aite. 1984. Rapid assessment of outerwood specific gravity in live trees using a pocket durometer. Can. J. For. Res. 14:145-150. 
Higa, A.T. 1963. Variacao de densidade basica de madera de Pinus elliottii var. elliottii e P. taeda. IPEF-Brasil 7:79-91.

J ozsa, L.A. y H. Brix. 1989. The effects of fertilization and thinning on wood quality of a 24-year-old Douglas-fir stand. Can. J. For. Res. 19:1137-1145.

Ladrach, W.E. 1984. Calidad de madera de Pinus patula Schl. et Cham. Informe de Investigación No. 92. Smurfit Cartón de Colombia Invest. Forestal. Cali, Colombia. 23 p.

Notivol, E., L.A. Gil y J .A. Pardos. 1992. Una metodología para la estimación de la densidad de la madera de árboles en pie y de su grado de variabilidad en Pinus pinaster Ait. Invest. Agrar. Sist. Rec. For. Vol. $1(1): 41-47$.

Smith, D.M. 1954. Maximum moisture content method for determining specific gravity of small wood samples. Forest Products Laboratory. Report 2014. Madison, Wi. 8 p.
Smith, D.M. 1955. Comparison of two methods for determining the specific gravity of small wood samples of second-growth Douglas-fir. Forest Products Laboratory. Report 2033. madison, Wi. 21 p.

Sprague, J.R., J.T. Talbert, J.B. J ett y R.L. Bryant. 1983. Utility of the pilodyn in selection for matured wood specific gravity in loblolly pine. Forest Sci. 29(4):696-701.

Valencia M., S. 1994. Variación genética de la densidad de la madera en Pinus patula y su relación con la velocidad de crecimiento. Tesis de Maestría en Ciencias. Colegio de Postgraduados. Montecillo, Méx. 108 p.

Zobel, B.J. y J.P. Van Buijtenen. 1989. Wood variation, its causes and control. Springer-Verlag. 363 p.

Zobel, B.J. y J. Talbert. 1984. A pplied forest trees improvement. J ohn Wiley and Sons. Nueva York. 505 p. 